\title{
First-principles prediction of surface wetting
}

Andersson, M. P.; Hassenkam, T.; Matthiesen, J.; Nikolajsen, L.; Okhrimenko, Denis V.; Dobberschütz, S.; Stipp, Susan L. S.

\section{Published in:}

Langmuir

Link to article, DOI:

10.1021/acs.langmuir.0c01241

Publication date:

2020

Document Version

Peer reviewed version

Link back to DTU Orbit

Citation (APA):

Andersson, M. P.., Hassenkam, T., Matthiesen, J., Nikolajsen, L., Okhrimenko, D. V., Dobberschütz, S., \& Stipp, S. L. S. (2020). First-principles prediction of surface wetting. Langmuir, 36(42), 12451-12459. https://doi.org/10.1021/acs.langmuir.0c01241

\section{General rights}

Copyright and moral rights for the publications made accessible in the public portal are retained by the authors and/or other copyright owners and it is a condition of accessing publications that users recognise and abide by the legal requirements associated with these rights.

- Users may download and print one copy of any publication from the public portal for the purpose of private study or research.

- You may not further distribute the material or use it for any profit-making activity or commercial gain

- You may freely distribute the URL identifying the publication in the public portal

If you believe that this document breaches copyright please contact us providing details, and we will remove access to the work immediately and investigate your claim. 


\title{
First-principles prediction of surface wetting
}

\author{
M.P. Andersson 1 ,*, T. Hassenkam², J. Matthiesen ${ }^{3}$, L. V. Nikolajsen ${ }^{1}$, D.V. \\ Okhrimenko $^{3}$, S. Dobberschütz ${ }^{3}$, S.L.S. Stipp ${ }^{4}$ \\ ${ }^{1}$ Department of Chemical and Biochemical Engineering, Technical University of \\ Denmark \\ ${ }^{2}$ Globe Institute, Section for Geobiology, University of Copenhagen, Denmark \\ ${ }^{3}$ Nano-Science Center, Department of Chemistry, University of Copenhagen, \\ Denmark \\ ${ }^{4}$ Department of Physics, Technical University of Denmark \\ * Corresponding author, martan@kt.dtu.dk
}

\begin{abstract}
We have developed a method for predicting the solvation contribution to solid-liquid interfacial tension based on density functional theory and the implicit solvent model COSMO-RS. Our method can be used to predict wetting behavior for a solid surface in contact with two liquids. We benchmarked our method against measurements of contact angle from water-in-oil on silica wafers and a range of self-assembled monolayers (SAMs) with different composition, ranging from oil-wet to water-wet. We also compared our predictions to literature data for wetting of a polydimethylsilane surface. By explicitly including deprotonation for the silica surfaces and carboxylic acid self-assembled monolayers, very good agreement was obtained with experimental data for nearly all surfaces. Poor agreement was found for amine-terminated SAMs, which could be a result of both method and model insufficiencies and impurities known to be present for such surfaces. Solid-liquid interfacial tension cannot be measured directly, making predictions such as from our method all the more important.
\end{abstract}

Keywords: solid-liquid interfacial tension, surface energy, contact angle, density functional theory, COSMO-RS

\section{Introduction}

The solid-liquid interfacial tension (IFT) is a determining factor for many physical and chemical phenomena. If a solid is in contact with more than one liquid, the balance of the IFTs, together with the surface structure, determine the wetting behavior of the liquids on the solid. This in turn determines e.g. how much oil can be recovered from a reservoir or how clean the water in an aquifer can become after being contaminated with organic compounds. The solid-liquid IFT not only determines solid wettability towards fluids ${ }^{1}$ but it also strongly influences the rate of mineral formation on organic solids ${ }^{2}$. Heterogeneous nucleation of minerals on an organic template is considered to be a limiting mechanism for how organisms control biomineralisation $^{2-3}$. Heterogeneous nucleation of a mineral on a surface is also the first step in unwanted mineral scaling in industrial processes such as cooling systems, geothermal energy production ${ }^{4}$ and oil recovery ${ }^{5}$. In a supersaturated solution, when a mineral grows, the solid-liquid IFTs for each of the mineral surface orientations ultimately determines the shape of the formed crystal. Therefore, finding the right set of IFT modifiers and predicting the ensuing surface energies accurately would allow 
design of nanoparticle shape and size. Sometimes, the solid-liquid surface free energy is termed the interfacial tension. In this paper, the terms are used synonymously.

Unfortunately, the solid-liquid IFT cannot be measured directly using existing techniques. At most, only differences in solid-liquid IFT can be determined, e.g. via contact angle measurements of one or two phase wetting on a solid ${ }^{6}$. The IFT can, on the other hand, be calculated using molecular modelling, e.g. using molecular dynamics for self-assembled monolayers of organic molecules (SAMs) on calcite ${ }^{7}$ and for the ice-water interface ${ }^{8}$. Density functional theory or other first principles methods have also been used to predict solid-vacuum IFT on crystalline solids. It has been shown that the choice of the method plays a large role in the validity of the predicted surface free energy of the termination of a solid in vacuum ${ }^{9}$.

In this paper, we present a method for predicting solid-liquid IFT based on the COSMO-RS model for liquid-liquid IFT ${ }^{10}$. Our method is able to predict the solvation contribution to the total solid-liquid IFT so it would be useful for predicting wetting phenomena, where only the differences between the solvents enter into the equations. Our results are limited to flat surfaces and only consider the contribution of surface chemistry to wetting but our results could be combined with knowledge about the surface structure to predict wetting for more complex surface geometries, where e.g. nanodimension domains have been shown to influence the whole surface free energy in a nonlinear fashion with respect to total composition ${ }^{11}$. Classical methods for predicting solid-liquid IFT require experimental input from contact angle measurements for a few different liquids on the particular solid, in order to provide empirical dispersive and polar contributions and finally solid-liquid IFT values. Our method is complimentary in that no experimental input is required in order to calculate the solid-liquid IFT, and the calculation can be made for liquids of arbitrary complexity. Our method is also significantly faster than molecular dynamics based methods by several orders of magnitude.

\section{Computational details}

The density functional theory calculations were performed using Turbomole, v $6.5^{12}$, the BP functional ${ }^{13}{ }^{14}$, the TZVP basis set ${ }^{15}$ for geometry optimizations and the COSMO implicit solvent model, ${ }^{16}$ using an infinite dielectric constant, which is required for subsequent COSMO-RS calculations ${ }^{17}$. An extra single point energy calculation with the TZVPD basis set was performed after the successful geometry optimization so we could use the BP_TZVPD_FINE_C30_1601 parameterization for the COSMO-RS calculations, all of which were performed using COSMOtherm ${ }^{18}$. The resulting molecular screening charge surface is called either a COSMO surface or a $\sigma$-surface, interchangeably in the literature. All parameterizations in COSMO-RS based on Turbomole calculations require the BP functional to be used.

The solid-liquid IFT method presented here is based on our recently developed method for liquid-liquid IFT ${ }^{10}$ and uses similar underlying equations, all of which are based on the flatsurf module of COSMOtherm ${ }^{18}$. Because COSMO-RS handles solvation via interaction of local screening charge surface interactions, the method allows for partial solvation at an interface. Flatsurf calculates the free energy of transferring a molecule from a bulk phase to a featureless interface via the partition function of all possible positions and orientations of the molecule at that interface. 
Analogous with our liquid-liquid method, we introduce a surface phase, which is allowed to have a different composition than the bulk liquid (the composition of the solid phase is kept unchanged throughout the calculation). One main difference between the liquid-liquid and solid-liquid methods lies in how the free energies of transfer are calculated (Figure 1). Because COSMO-RS interactions within the solid are meaningless, we had to change how some of the contributions to the total IFT were calculated from the two interfaces. The interface between the bulk liquid and the surface phase was handled in the same way as in the standard liquid-liquid IFT method but for the interface between the surface phase and the solid, the COSMO-RS interaction, had to be calculated with the surface phase as the "bulk" phase. Also, because the free energy contributions to the IFT are two sided at each surface phase interface, we had to divide the contributions from the surface phase-solid interface by 2 to take into account that only one side contributes to the IFT. This is shown as a smaller $\Delta \mathrm{G}$ arrow in Figure 1 and adds a factor of $1 / 2$ to Equation 2. The contribution from the solid side is not taken into account in our method but could potentially be accounted for by a separate calculation of the surface energy of the solid in vacuum. The equations required to calculate the solid-liquid IFT are:

$$
\begin{aligned}
I F T_{A S} & =\sum_{i} \frac{\theta(i)\left[G_{t o t, A \rightarrow S}(i)-R T \ln \left(x_{A}(i)\right)+R T \ln (\theta(i))\right]+x_{A}(i) G_{t o t, A \rightarrow S}(i)}{2 A_{a v, A \rightarrow S}(i)} \text { and } \\
I F T_{B S} & =\sum_{i} \frac{\theta(i)\left[G_{t o t, S \rightarrow B}(i)\right]}{2 A_{a v, S \rightarrow B}(i)} .
\end{aligned}
$$

$\theta(i)$ is the mole fraction of component $i$ in the surface phase $\mathrm{S}, G_{t o t, A \rightarrow S}(i)$, is the free energy of transferring molecule $\mathrm{i}$ from phase A to the surface phase $\mathrm{S}, x_{A}(i)$ is the mole fraction of component $\mathrm{i}$ in phase $\mathrm{A}$ and $A_{a v, A \rightarrow S}(i)$ is the average crosssection area for the molecule $i$ at the interface between phases $A$ and $\mathrm{S}$. The total COSMO-RS IFT of the solid-liquid interface is then:

$I F T=I F T_{A S}+I F T_{B S}$

An additional modification, which does not show in the equations, was required for the (surface phase)-solid interface, which stems from the fact that the adhering molecule cannot penetrate into the solid. We constrained the partition sum in the flatsurf calculations by imposing a maximum penetration depth (Figure 2) of $3.0 \AA$ in all flatsurf calculations at the surface phase-solid interface, excluding all configurations with a larger penetration depth. This constraint, which affects the $\mathrm{G}_{\text {tot }}$ values in Equations (1) and (2), was imposed via the maxdepth keyword in COSMOtherm and enabled some parts of the molecule in solution to interact with the solid interface, but not all. This simulates the situation where a part of the molecular surface could be in contact with the solid, where the rest of the molecule, not interacting with the solid, would interact with the surrounding liquid, mimicking adsorption. Too large values of maxdepth does not make physical sense, as during adsorption, not all parts of a molecule can be in contact with the surface at the same time. We therefore investigated maxdepth values of 1, 2, 3 and $4 \AA$ for five SAM systems $\left(-\mathrm{CH}_{3},-\mathrm{C}_{6} \mathrm{H}_{5},-\mathrm{NH}_{2},-\mathrm{COOH}\right.$ and $-\mathrm{OH}$, Table $\mathrm{S} 1$ in supporting information).Values of 2 and 3 and $4 \AA$ gave the lowest mean absolute deviation with rather similar results, showing that our IFT calculations are not very sensitive to the choice of maxdepth. We chose to use $3 \AA$ throughout the paper because it was the 
middle value of the three, as well as being most in agreement with the experimental results for the SAM - OH system, where no deprotonation is occurring and no contamination is known. The calculation script is freely available in GitHub at github.com/LasseNikolajsen/ift from lle.

Our method does not predict the absolute solid-liquid IFT, only the contribution from the liquid phase - the solvation part. In the case of e.g. a crystalline solid, the termination energy in vacuum would have to be added, from experiment or from e.g. DFT calculations, and these contributions are generally quite large. The crucial aspect for this paper is that for wetting phenomena, this vacuum contribution cancels as long as the solid surface is the same in the two liquids. For a system with a surface, s, in contact with oil, o, and water, w, the contact angle of a water-in-oil droplet on a flat, featureless surface is described by Young's equation:

$\cos \theta=\frac{\gamma_{o s}-\gamma_{w s}}{\gamma_{o w}}$

The $\gamma_{o s}$ is the solid-liquid IFT between oil and solid, the $\gamma_{\mathrm{ws}}$ is the solid-liquid IFT between water and solid and $\gamma_{\text {ow }}$ is the liquid-liquid IFT between oil and water.

Because the total IFT can be viewed as a sum of the surface energy for forming the surface in vacuum plus the COSMO-RS solvation contributions that act on the solid surface, the solid-liquid IFTs or surface free energies in Eqn 4 can be written:

$\gamma_{w s}=\gamma_{\text {solid formation,vacuum }}+\gamma_{w s, \text { COSMO-RS }}$

$\gamma_{o s}=\gamma_{\text {solid formation,vacuum }}+\gamma_{\text {os, COSMO-RS }}$

Because the solid formation free energy is the same in both cases (no solvent), the predicted contact angle is:

$\cos \theta=\frac{\gamma_{O S, \operatorname{COSMO}-R S}-\gamma_{w s, C O S M O-R S}}{\gamma_{o w}}$.

Exactly which terms would constitute the non-COSMO-RS interactions to the IFT is not the focus of this paper. We conjecture that for a crystalline solid, the contributions would include the vacuum surface termination energy and for a crystalline solid with a strongly adsorbed layer such as the SAMs in this paper or the first monolayer of water on strongly hydrated surfaces, such as calcite ${ }^{19}$, the contributions would include the surface termination energy and the adsorption energy of the layer (per unit area). A detailed investigation of this topic is left for the future.

In order to take into account that only the end groups of the SAM are exposed to the liquid phase, we used a weighting factor of 0 for the spacer chain of the SAM in the COSMOtherm calculations to eliminate solvation interaction. In this way, we were able to model a system, where the spacer chain is not exposed to the solvent, while the end group is. We used the following molecules to model the various SAM components: n-decane, acetic acid, 1-propylamine, 1-octanol and benzene. The partial COSMO surfaces are shown in Figure 3. By using the COSMO surface of the functional group only, no noticeable difference was observed for a different alkyl chain length. Two conformers related to the $\mathrm{OH}$ group geometry for acetic acid and 1- 
octanol were included in the calculations but only one conformer for each molecule is shown in Figure 3.

We used a model for the polydimethylsilane (PDMS) surface, based on the structure shown in Figure 4. The formula is $\left(\mathrm{CH}_{3}\right)_{3} \mathrm{Si}-\left(\mathrm{OSi}\left(\mathrm{CH}_{3}\right)_{2}\right)_{3}-\mathrm{OSi}\left(\mathrm{CH}_{3}\right)_{3}$. As is commonly done in COSMO-RS modelling of polymers, we excluded the two end monomers in the definition of the COSMO surface.

Two simple models for the silica surface were used, a silica monomer and a silica tetramer, both shown in Figure 5, together with the monomer model for the deprotonated surface groups.

For mixed SAMs, we assumed no island formation. The water droplet, for which the contact angle was measured, was thus much larger than the spatial scale over which chemical heterogeneity would be observed. As a consequence, the COSMO-RS solvation IFT was calculated by simply weighting over the relative composition of the constituents A and B (the COSMO-RS subscript is omitted):

$\gamma_{w s}=x_{S A M, A} \cdot \gamma_{w s, A}+x_{S A M, B} \cdot \gamma_{w s, B}$

where $x_{S A M, A}$ represents the mole fraction of Component $\mathrm{A}$ in the surface of the self assembled monolayer.

In the case where surface chemical heterogeneities are larger than the extent of the probing liquid drop, a range of contact angles would be expected, which would be different, depending on where on the surface the droplet was located. By probing the chemical composition of the surface with enough spatial resolution, local contact angles could be predicted and compared with calculations for the various surface compositions.

\section{Charged solid surfaces}

While COSMO-RS in principle requires neutral phases for thermodynamic consistency, it is possible to override this check using the ignore_charge keyword. While this makes little sense for bulk liquid phases, we made calculations for charged surfaces by performing calculations using ions as models for the solid. The thermodynamic consistency is not lost in this case because there is no mixing of the solid phase into the liquid and surface phases, keeping all liquids neutral. In principle, this neglects the countercharge of the layer adjacent to the surface but because our method does not provide a size for the surface phase, it is difficult to define the proper concentration of the counterions. Therefore, we choose to take the simplest approach, by keeping the surface phase neutral, also when interacting with a charged surface. The only contribution comes from the flatsurf calculations, where molecules from the surface phase interact with the solid phase. For determining the contact angle when some sites were deprotonated, this correction was only made for the water phase. We assumed that no deprotonation would occur in the nonpolar oil phase. For any partly deprotonated surface in water, a linear interpolation of the IFT, according to Equation 7, was used.

If some surface functional groups can protonate or deprotonate, such as $-\mathrm{COOH}$, the free energy of the system can be decreased by allowing some groups to become 
charged. This in turn affects the wetting properties and we can account for this in the COSMO-RS interactions. For $\mathrm{pH}$ above the surface $\mathrm{pK}_{\mathrm{a}}$, there is a chemical driving force for deprotonation of such functional groups. Because the deprotonated groups are stuck on a surface, a counter force occurs from electrostatic repulsion between surface charges, which is screened in saline aqueous solutions. We have developed a mean field model, based on Yukawa potentials for the electrostatic interactions (simple screened electrostatics), which provides the change in free energy from deprotonation as a function of the fraction of deprotonated groups, $\theta, \mathrm{pH}$ and ionic strength:

$\Delta G=2.3 R T \theta\left(p K_{a}^{\text {surf }}-p H\right)+\frac{1}{2} \frac{6(\theta e)^{2}}{4 \pi \varepsilon_{0} \varepsilon_{r} d_{0}} \frac{1}{e^{\left(\frac{d_{0}}{\lambda_{d}}\right)}-1}-R T \theta \ln \left(\frac{1}{\theta}\right)$

$p K_{a}^{\text {surf }}$ represents the $\mathrm{pK}_{\mathrm{a}}$ of the functional group at the interface, $d_{0}$, the nearest neighbor distance between surface groups in a hexagonal lattice and $\lambda_{d}$, the Debye length (which can be calculated from the ionic strength) and $e$ is the elementary charge. The first term in Equation 8 is the chemical driving force for deprotonation, the second term is the screened electrostatic repulsion and the last term is the configurational entropy for deprotonating a fraction, $\theta$, of the surface in a mean field model. The derivation of the electrostatic repulsion term is based on an approximation via a geometric sum and is described in the Supporting Information. By minimizing the free energy for a given surface $\mathrm{pK}_{\mathrm{a}}$, solution $\mathrm{pH}$ and ionic strength, the degree of deprotonation, $\theta$, can be determined. $\theta$, in turn, determines the balance of COSMORS interactions between the charged and neutral models for the surface by means of Equation 7. A detailed comparison between the mean field model and an explicit treatment of various deprotonation configurations as well as experiments is in progress. In short, a full statistical mechanical description of summation of explicit configurations of deprotonated surfaces gives quite similar results to the mean field model presented here, which is also in qualitative agreement with Atomic Force Microscopy measurements of forces between SAM - $\mathrm{COOH}$ surfaces at varying $\mathrm{pH}$.

\section{Experimental details}

\section{Preparation of self assembled monolayers}

Flat gold substrates for making self assembled monolayers (SAMs) were prepared using the template stripping approach ${ }^{20}$. One hundred nanometer gold films on four inch Si wafers from Tebu-bio/Platypus Technologies were used. A $2 \mu \mathrm{L}$ drop of EpoTek 353ND epoxy glue (Thorlabs) was put on a clean Si wafer measuring $5 \mathrm{~mm} \times 7$ $\mathrm{mm}$. The Si wafer was placed with its glue face down on the gold film without applying any additional pressure. The sandwich structure was put on a hot plate at 120 ${ }^{\circ} \mathrm{C}$ for 30 min to cure the epoxy glue. The small Si wafer/Epo-Tek/gold structure was stripped from the large Si wafer by gently pushing the edge of a scalpel blade under one corner of the small $\mathrm{Si}$ wafer.

The SAMs were formed by immersing the Au coated substrates into a 4-5 mM solution of thiol terminated molecules for at least $24 \mathrm{~h}$ at room temperature. Thiol solutions consisting of just one type of molecule were made by dissolving $\mathrm{HS}\left(\mathrm{CH}_{2}\right)_{10} \mathrm{CH}_{3}\left(98 \%\right.$, Sigma Aldrich), $\mathrm{HS}\left(\mathrm{CH}_{2}\right)_{10} \mathrm{COOH}(99 \%$, Sigma Aldrich), $\mathrm{HS}\left(\mathrm{CH}_{2}\right)_{11} \mathrm{OH}\left(98 \%\right.$, Sigma Aldrich) or $\mathrm{HS}\left(\mathrm{CH}_{2}\right)_{10 \text { or } 11} \mathrm{NH}_{2}(>90 \%$, Dojindo Molecular 
Technologies) in 99.8\% pure ethanol (HPLC grade, Sigma Aldrich). Solutions for mixed SAMs were made by mixing two of the solutions. The composition for a mixed SAM is reported using the mole fraction of the thiols in the solution. The thiol terminated molecules order themselves on the gold surfaces into a dense, self assembled monolayer with the sulfur atoms bound to the gold and the $-\mathrm{CH}_{3}$ or $\mathrm{COOH}$ group pointing away from the surface ${ }^{21}$. After removal from the solution, the samples were rinsed with ethanol and dried in a flow of clean $\mathrm{N}_{2}$.

\section{Materials for the silica wafer experiments}

We used boron doped silicon wafers (Silicon Material Inc.) that had been chemically polished to a final surface roughness $<2 \AA$. The initial diameter of the wafers was 150 $\mathrm{mm}$, thickness was $675 \pm 15 \mu \mathrm{m}$ and the surface $(100 \mathrm{~nm})$ had been wet oxidized so they were $\mathrm{SiO}_{2}$. The wafers were cut into $10 \times 10 \mathrm{~mm}$ squares by the manufacturer. The silica wafers were treated in a UV-ozone cleaner for $30 \mathrm{~min}$, rinsed with ultrapure deionised water (MilliQ; MQ) and blown dry with a pure $\mathrm{N}_{2}$ gas stream. Cleaned silica wafers were used for contact angle measurements straight after the cleaning procedure or after being placed in n-dodecane for 20 hours. The n-dodecane on the silica wafer was blown dry with a $\mathrm{N}_{2}$ stream and the contact angle was measured directly after that.

\section{Measurement of water contact angles on solid substrates}

\section{Self assembled monolayers}

The SAM coated substrates were carefully put at the bottom of a quartz cuvette filled with n-dodecane ( $>99 \%$, Sigma Aldrich). A 1-5 $\mu \mathrm{L}$ droplet of $100 \mathrm{mM} \mathrm{NaCl}$ at $\mathrm{pH}$ 5.5 was carefully placed on the SAM surface using a Hamilton syringe. An image of the droplet was taken immediately using a USB microscope. Typically a second droplet was placed without touching the first droplet. The USB microscope was used to capture an image of the second droplet immediately after placement. The contact angle of the saline water droplet was determined using the contact angle plugin for Image J by Marco Brugnara ${ }^{22}$. A photo from such a measurement is shown in Figure 6 .

\section{Silica wafers}

Contact angle images were taken using DNT DigiMicro Profi USB camera and captured using the MicroCapturePro software. The water droplet was put on the silica surface in n-dodecane using a $10 \mu \mathrm{L}$ pipette. The volume of the water droplets was equal to $1 \mu \mathrm{L}$ and duplicate measurements were made on the right and left sides of the droplet. Contact angles were determined using the ImageJ software.

\section{Results and Discussion}

The predicted and measured contact angles for water in n-dodecane on a range of SAMs are shown in Figure 7 and Table 1. Our predictions for the contact angle fit the experimental results reasonably well (generally within $\sim 30^{\circ}$ ) for surfaces with quite different composition, ranging from an oil-wet surface consisting of $-\mathrm{CH}_{3}$ end groups to an almost completely water wet surface with -OH end groups. In particular, the 
qualitative behavior is very well described and the trends are clearly reproduced, including the observed difference between aliphatic and aromatic SAMs. The mean absolute deviation was $26^{\circ}$, If we removed the $-\mathrm{NH}_{2} \mathrm{SAM}$ from the comparison, the mean absolute deviation was only $15^{\circ}$. This corresponds to an error in solid-liquid IFT of $\sim 2 \mathrm{mN} / \mathrm{m}$ for contact angles near 0 or $180^{\circ}$ and $\sim 12 \mathrm{mN} / \mathrm{m}$ for contact angles around $90^{\circ}$. This deviation between calculations and experimental results is generally larger than what we found for our liquid-liquid IFT method ${ }^{10}$, where it was $\sim 3 \mathrm{mN} / \mathrm{m}$. There could be several reasons for this. First, our solid-liquid interface method depends on two additional approximations: the use of maxdepth to model the surface as a solid and the use of partial COSMO surfaces to model the surface composition of the SAMs. The choice of maxdepth influenced the contact angles by about 10 degrees (Table S1). Part of the deviation between experiments and predictions could also arise from our assumptions about the surface composition for the two systems with the largest deviations, namely for $-\mathrm{COOH}$ and $-\mathrm{NH}_{2}$, which we will now discuss in more detail.

For - $\mathrm{COOH}$, no deprotonation of the surface was considered for constructing Figure 7. The $\mathrm{pH}$ of the solutions we worked with was 5.5, meaning that at least part of a carboxylic acid surface would be deprotonated, which would explain the overestimation of the contact angle made by the method. A charged surface would result in a more water wet surface. We investigated the effect of allowing some surface $-\mathrm{COOH}$ groups to deprotonate into - $\mathrm{COO}^{-}$. We used acetate as the model for the deprotonated surface to obtain the IFT and assumed a specific ratio for deprotonated surface acid groups, with Equation 7. The results are shown in Figure 8 for $0 \%, 22 \%$ and $100 \%$ deprotonated surface groups. Indeed the water wetness of the surface increased when the surface was charged, with $22 \%$ deprotonation agreeing well with the experiments. The reason for the increased water wetness is that the IFT between the charged surface and water becomes much more negative than for the corresponding neutral composition (Table 2). The $\mathrm{pH}$ was 5.5 in our measurements and the $\mathrm{pK}_{\mathrm{a}}$ for- $\mathrm{COOH}$ at the SAM-water surface was predicted to be $4.7^{23}$ for a small degree of deprotonation. Based on normal aqueous equilibria, the degree of deprotonation would thus be $>50 \%$ but when confined at an interface, the chargecharge interactions at the surface must be taken into account. We have implemented a model for estimating the degree of deprotonation at a surface using the mean field model in Eq. 8, requiring only the pKa of the surface group and the ionic strength of the solution. For a solution of $\mathrm{pH}=5.5$, ionic strength $=0.1 \mathrm{M}$ and $\mathrm{pKa}=4.7$, the model predicts the degree of deprotonation to be $22 \%$, which fits very well with experiments (Figure 8). Qualitatively, the charge-charge repulsion between deprotonated acid groups would disfavor deprotonation, so we would expect the degree of deprotonation to be lower than estimated from aqueous equilibria, which is consistent with our estimate of $22 \%$ based on the theoretical model. For surfaces with functional groups that can deprotonate, computational methods that can predict the degree of deprotonation would allow for a more accurate prediction of the solid-liquid IFT.

In contrast with behaviour of the $-\mathrm{COOH}$ surface, the $-\mathrm{NH}_{2}$ surface is less water wet in experiments than our predictions. This could be a result of the high reactivity of $\mathrm{NH}_{2} \mathrm{SAMs}^{24}$, which in XPS, yields a peak for water even in ultrahigh vacuum. Thus the $-\mathrm{NH}_{2}$ surface is quite prone to adsorption of contaminants, which would generally make the surface more oil wet. Such adsorption was not taken into account in our 
predictions and neither was any protonation. If the concentration and nature of the surface active compounds were known, they could in principle be taken into account in the calculations. However, our focus in this work was to develop the predictive methods.

We also predicted the solid-liquid IFT for another set of experimental interfacial energies, derived from contact angles ${ }^{25}$. The experiments used a PDMS surface and tested the wetting behaviour of a range of organic molecules and water. The comparison between our predictions and the experimental data is presented in Figure 9 and Table 3 . The correlation was quite good for all liquids, with a very low mean absolute deviation of $4 \mathrm{mN} / \mathrm{m}$ between our predictions and the experiments.

A comparison between predicted and experimental contact angles for water in $n$ dodecane on silica is shown in Table 4. For a completely neutral surface, the results from the experiments suggest a more water wet surface than our predictions. The experimental $\mathrm{pH}$ was $\sim 5.5$. This is higher than the point of zero charge for silica, which lies around $\mathrm{pH}=2-3{ }^{26}$. Therefore, some of the surface -SiOH groups would be deprotonated, giving rise to negative surface charge, as for the -COOH SAM. By including more negatively charged groups, modelled by the deprotonated monomer, our predictions for $10 \%$ deprotonated groups agree with experimental contact angle in Table 4. The degree of deprotonation is less than for the acid SAM, which qualitatively agrees with a higher $\mathrm{pK}_{\mathrm{a}}$ for the $-\mathrm{SiOH}$ monomer than for the $-\mathrm{COOH}$ group. The $\mathrm{pKa}$ for the silica monomer was 8.1 from our calculations but the surface model is rather crude. In general, IFT predictions for inorganic surfaces should be considered qualitative until more sophisticated surface models have been explored. The silica surface that was exposed to n-dodecane was more oil-wet than the freshly ozone cleaned surface, which is likely caused by surface active contaminants in the ndodecane ${ }^{27}$. The contaminants accumulate at any interface between hydrophobic and hydrophilic surfaces, such as between n-dodecane and silica.

The comparison between experimental data and our predicted solid-liquid interfacial energy is very good for many, quite different systems, with one important caveat: A reasonable model of the solid surface is required and this is not always straightforward. Sometimes, the surface composition and structure are not known well enough, as for the silica surface and the $-\mathrm{NH}_{2} \mathrm{SAM}$. Finally, for surfaces, where functional groups exist, that can protonate or deprotonate, the charge state should be estimated as accurately as possible and taken into account in the calculations.

It is possible in some systems to get the values of the solid-liquid IFT from experiments, and when possible that is absolutely a good idea. The point with our computational method is that it's complimentary and can provide additional information. For example, when for most systems the experiments and calculations agree, large deviations can be a hint that the model system is not equal to the experimental system. Then, the model itself needs to be updated to provide better understanding of the real system, like the amine based SAMs, which have been shown in other experiments to be impossible to keep as pristine $-\mathrm{NH}_{2}$ surfaces (like the model). Even in vacuum, surface contaminations are observed. And second, the speed of the method could allow for computational screening of material properties, much faster than experiments could do.

\section{Conclusions}


We have developed a method for predicting the solvation contribution to solid-liquid interfacial tension, based on density functional theory and the COSMO-RS implicit solvent model. The method is particularly useful to predict the wetting behavior of two liquids in contact with a solid surface, where other contributions in addition to solvation cancel. We benchmarked our method against experimentally measured contact angles and interfacial energies and found that the method reproduced the influence of surface composition on wetting, for flat surfaces. The trends were captured by our method for hydrophobic to hydrophilic surfaces, including both organic surfaces and a silica surface. For two negatively charged surfaces, a $-\mathrm{COOH}$ self assembled monolayer and a silica surface, the predicted contact angles for water in oil were higher than from experiments, i.e. the surface was more water wet than the predictions, because deprotonation and surface charge were initially not taken into account. By including deprotonation of the surface, our contact angle predictions agreed with the experimental data. Because solid-liquid IFT is very difficult to measure, the availability of a predictive method is quite important. Our method could find application in designing surfaces with desired wetting properties and could help explain the influence of surface composition and structure on heterogeneous nucleation of minerals on organic substrates, such as in a biomineralization model system. The method could also be combined with other calculations for the vacuum surface energy, to obtain the total IFT, which would be required to model growth morphology where the IFT of several solid surfaces becomes relevant.

\section{Acknowledgements}

The work was part of the Nano-Sand Project, funded by BP Exploration Operating Company Ltd. through the ExploRe Program. We also acknowledge support from the W-EOR Project, funded by Maersk Oil Research and Technology Centre. Access to computing resources was provided by the Danish Center for Scientific Computing (DCSC), which has since changed name to the Danish e-Infrastructure Cooperation (DeIC). A small contribution came from the EPSRC (Engineering and Physical Sciences Research Council) of the U.K., through the Frame Project, MIB (EP/I001514/1). This work also partly received financial support from the Greater Copenhagen Food Innovation project (CPH-Food). We also thank Professor Georgios Kontogeorgis for valuable scientific discussions on classical modeling of solid-liquid IFT.

Table 1. Experimental and predicted water-in-oil contact angles on a range of self assembled monolayers (SAM). The average standard deviation for the measurements was $6^{\circ}$.

\begin{tabular}{l|ccc} 
Composition of the SAM & $\begin{array}{l}\text { Experimental } \\
\text { contact angle }\left[^{\circ}\right]\end{array}$ & $\begin{array}{l}\text { Calculated } \\
\left.\text { contact angle [ }{ }^{\circ}\right]\end{array}$ & $\begin{array}{l}\text { Calculation - } \\
\text { experiment }\left[^{\circ}\right]\end{array}$ \\
\hline $100 \%-\mathrm{CH}_{3}$ & 164 & 180 & 16 \\
$22 \%-\mathrm{COOH}, 78 \%-\mathrm{CH}_{3}$ & 130 & 136 & 6 \\
$44 \%-\mathrm{COOH}, 56 \%-\mathrm{CH}_{3}$ & 100 & 114 & 14 \\
$72 \%-\mathrm{COOH}, 28 \%-\mathrm{CH}_{3}$ & 68 & 90 & 22 \\
$100 \%-\mathrm{COOH}$ & 33 & 66 & 33 \\
$22 \%-\mathrm{OH}, 78 \%-\mathrm{CH}_{3}$ & 139 & 129 & -10
\end{tabular}


$48 \%-\mathrm{OH}, 52 \%-\mathrm{CH}_{3}$

$100 \%-\mathrm{OH}$

$22 \%-\mathrm{NH}_{2}, 78 \%-\mathrm{CH}_{3}$

$\begin{array}{lll}155 & 120 & -35\end{array}$

$48 \%-\mathrm{NH}_{2}, 52 \%-\mathrm{CH}_{3}$

140

$82-58$

$72 \%-\mathrm{NH}_{2}, 28 \%-\mathrm{CH}_{3}$

90

$100 \%-\mathrm{NH}_{2}$

75

$44-46$

$100 \%-\mathrm{C}_{6} \mathrm{H}_{5}$

123

0

Mean absolute deviation

143

Maximum deviation

Table 2. Solid-liquid IFT predictions for our methods used to calculate the contact angles for the SAMs and the silica surface.

\begin{tabular}{l|cc} 
Surface model (Figures 3 and 4) & $\begin{array}{c}\text { Predicted water/surface } \\
\text { IFT [mN/m] }\end{array}$ & $\begin{array}{l}\text { Predicted n-dodecane } \\
\text { /surface IFT [mN/m] }\end{array}$ \\
\hline$-\mathrm{CH}_{3}$ & 59 & 5 \\
$-\mathrm{C} 6 \mathrm{H} 5$ & 48 & 7 \\
$-\mathrm{NH}_{2}$ & -53 & 20 \\
$-\mathrm{COOH}$ (conformer 1) & -0 & 17 \\
$-\mathrm{COOH}$ (conformer 2) & -5 & 18 \\
$-\mathrm{OH}($ conformer 1) & -22 & 17 \\
$-\mathrm{OH}($ conformer 2) & -26 & 20 \\
$\mathrm{Si}(\mathrm{OH})_{4}$ & -3 & 30 \\
$\mathrm{Si}-\mathrm{tetramer}$ & -6 & 24 \\
$\mathrm{HCOO}-$ & -145 & 2 \\
$\mathrm{Si}(\mathrm{OH})_{3} \mathrm{O}-$ & -356 & -9
\end{tabular}

Table 3. Experimental and predicted IFT of various liquids on a PDMS surface. All values are in $\mathrm{mN} / \mathrm{m}$.

\begin{tabular}{l|llc} 
Liquid & $\begin{array}{l}\text { Experimental } \\
\text { IFT } \\
\text { PDMS-liquid }\end{array}$ & $\begin{array}{l}\text { Predicted } \\
\text { IFT }\end{array}$ & $\begin{array}{l}\text { Predicted - } \\
\text { Exp. IFT }\end{array}$ \\
& & & \\
\hline nitromethane & 8.5 & 13 & 5 \\
DMSO & 10.2 & 11 & 1 \\
ethylene glycol & 17.9 & 15 & -3 \\
$20 \%$ ethanol in water & 26.0 & 31 & 5 \\
Water & 40.5 & 37 & -4 \\
Mean absolute deviation & & & 4
\end{tabular}

Table 4. Experimental and predicted contact angle of water in n-dodecane on silica. 


\begin{tabular}{l|c} 
System & Contact angle $\left[^{\circ}\right]$ \\
\hline DFT/COSMO-RS for silica monomer & 51 \\
DFT/COSMO-RS for silica tetramer & 54 \\
DFT/COSMO-RS for silica monomer, 3\% deprotonated & 36 \\
DFT/COSMO-RS for silica monomer, 6\% deprotonated & 6 \\
DFT/COSMO-RS for silica monomer, $>6 \%$ deprotonated & 0 \\
Experiment on silica wafer, measured directly after ozone cleaning & 29 \\
Experiment on silica wafer, measured after 20 hours in dodecane & 40
\end{tabular}

Liquid/Liquid

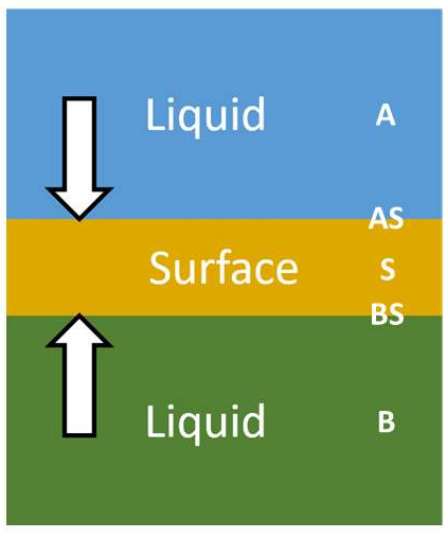

Liquid/Solid
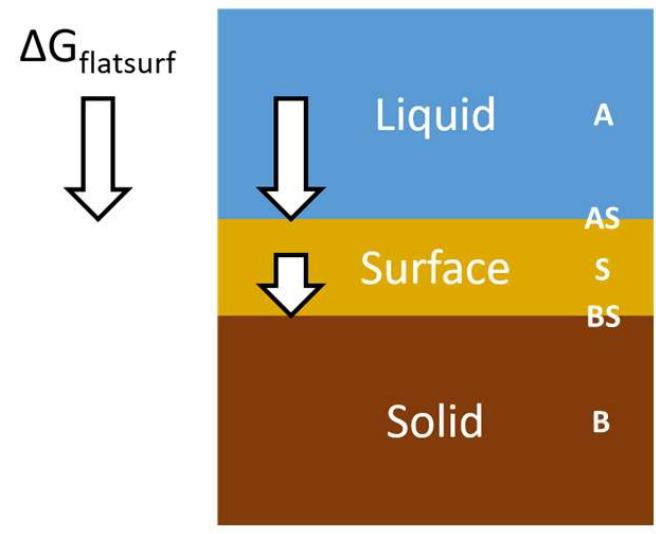

Figure 1. Schematic depiction of how the free energy contributions in the solid-liquid IFT method presented in this paper compare with the original liquid-liquid IFT method ${ }^{10}$. The Surface phase $\mathrm{S}$ is a fictious phase which can have a different composition than either of the liquid phases, depending on if surface active molecules are present or not. The white arrows represent the free energy contributions calculated using COSMO-RS for transferring a molecule from one phase (Liquid or Surface start of arrow) to an interface between two phases (end of arrow). 


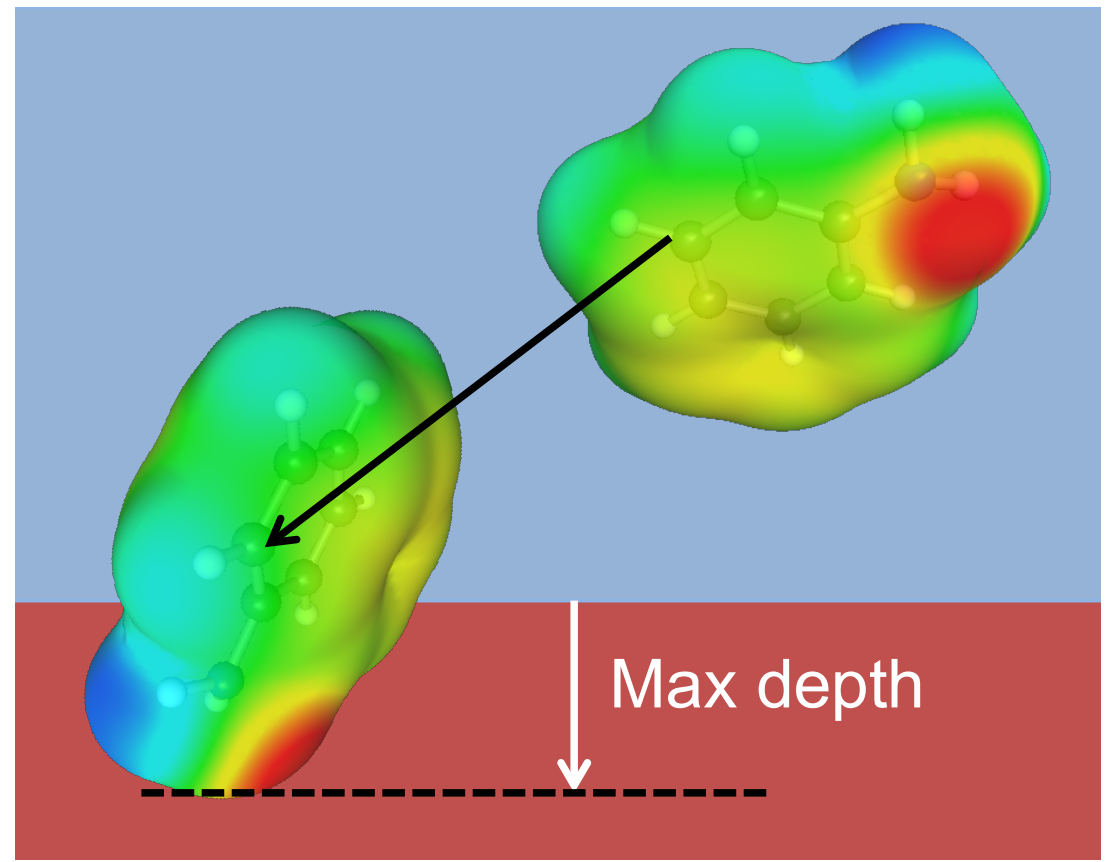

Figure 2. Schematic diagram showing the constraint in the flatsurf calculations for interactions of molecules from the surface phase (blue) with the solid (red-brown). The molecule is only allowed to penetrate to a certain depth into the solid, to make sure that only part of the molecule in the surface phase can interact with the solid.
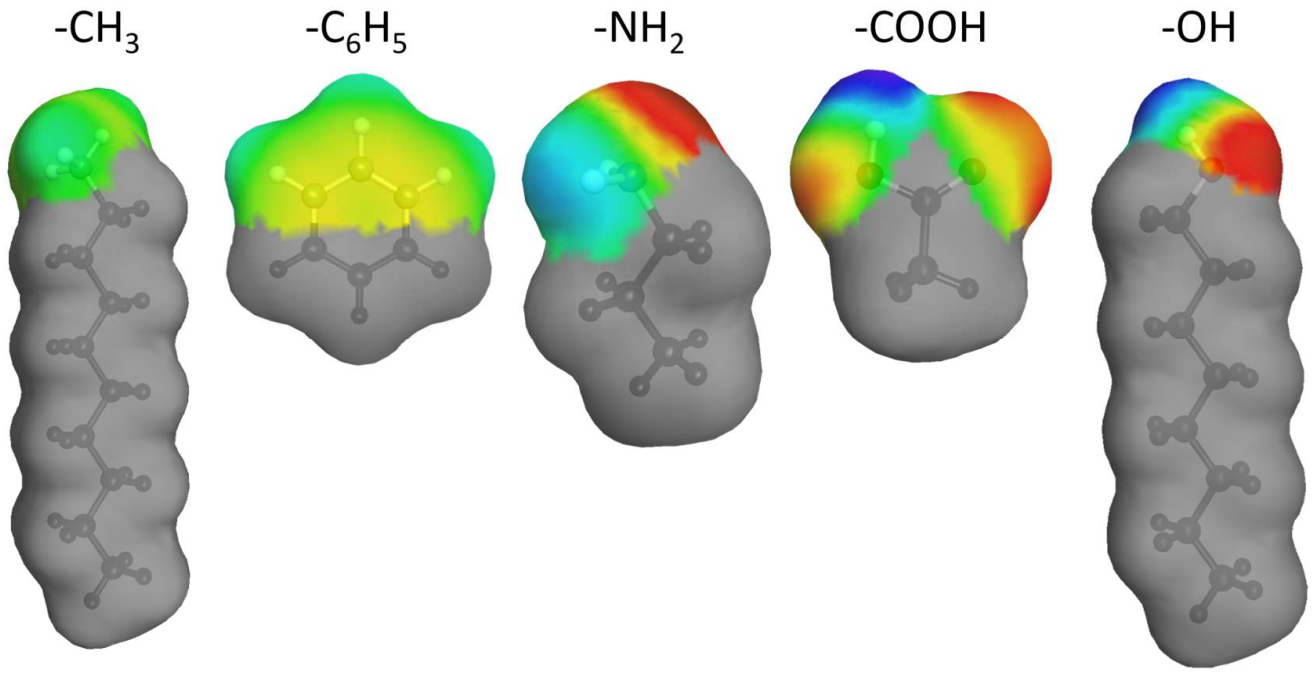

Figure 3. COSMO surfaces used to model the surface composition of the self assembled monolayers. 

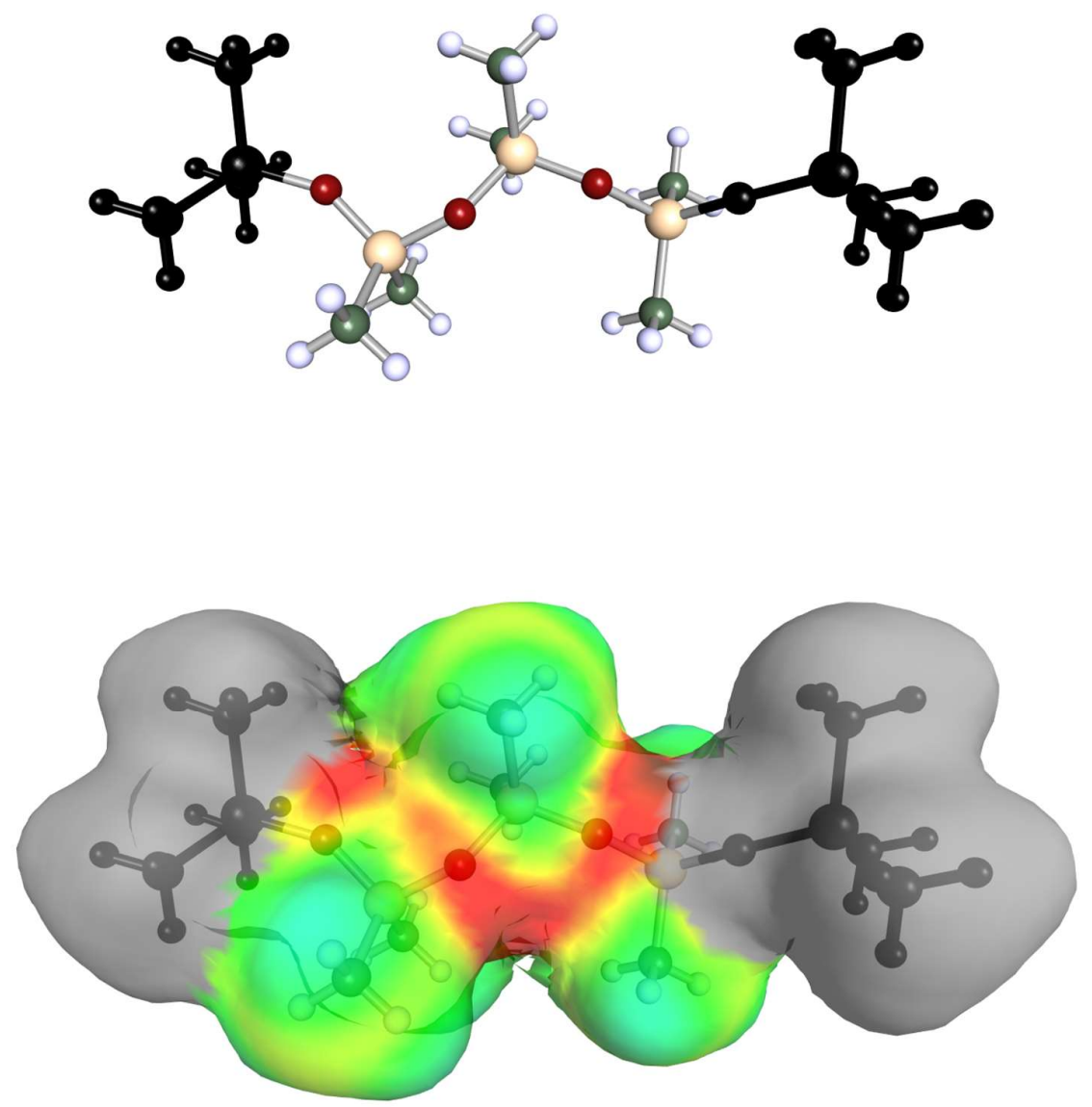

Figure 4. The model molecule used for polydimethylsilane PDMS (top) with the COSMO surface used for the molecule (bottom). The end groups were not used, in order to better model a polymer chain. 

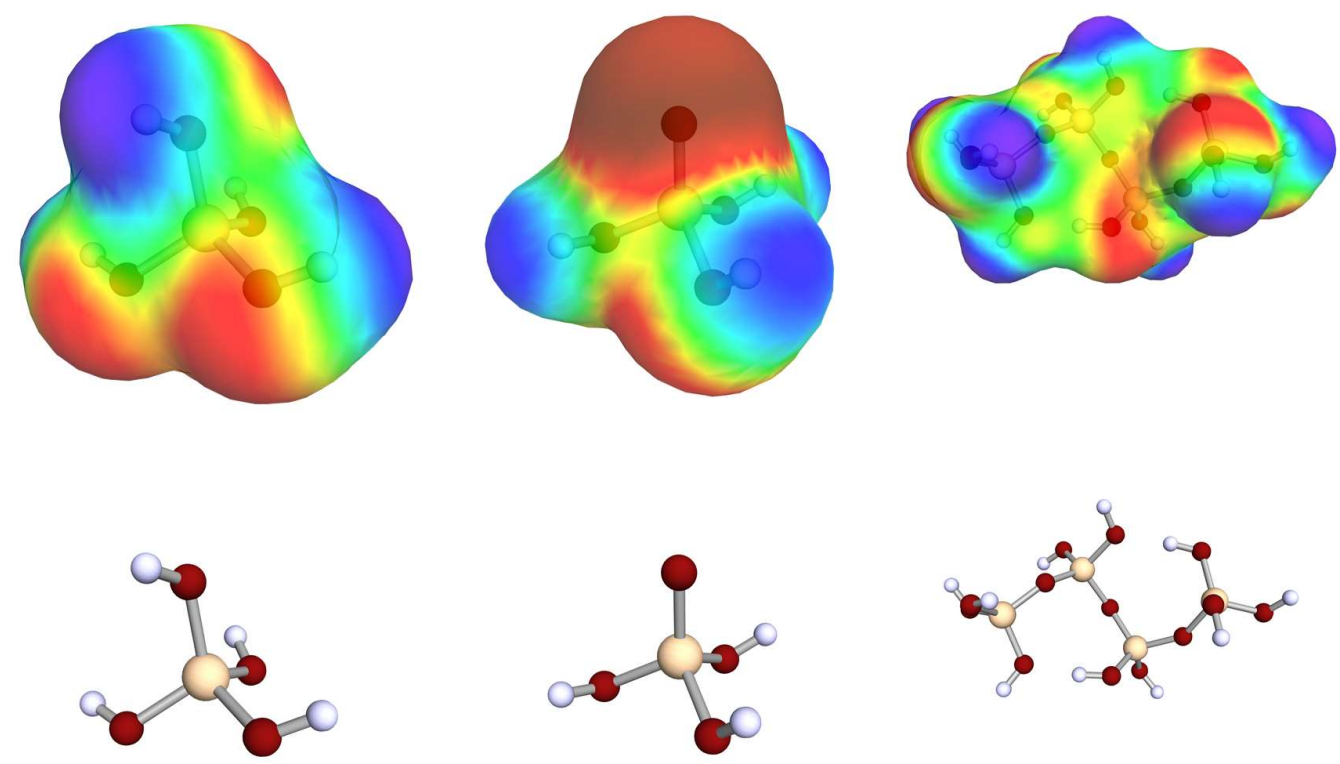

Figure 5. COSMO surfaces (top) and molecule geometries (bottom) used to model the silica surfaces. Left: Silica monomer, $\mathrm{Si}(\mathrm{OH})_{4}$. Middle: Singly deprotonated silica monomer, $\mathrm{Si}(\mathrm{OH})_{3} \mathrm{O}^{-}$. Right: Silica tetramer, $\mathrm{Si}_{4}(\mathrm{OH})_{13}$.

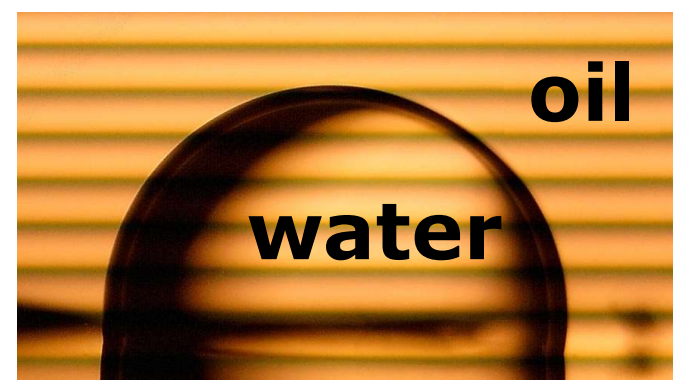

Figure 6. Camera view of a water droplet in dodecane used for the contact angle measurements. 


\section{Experimental and predicted contact angles}
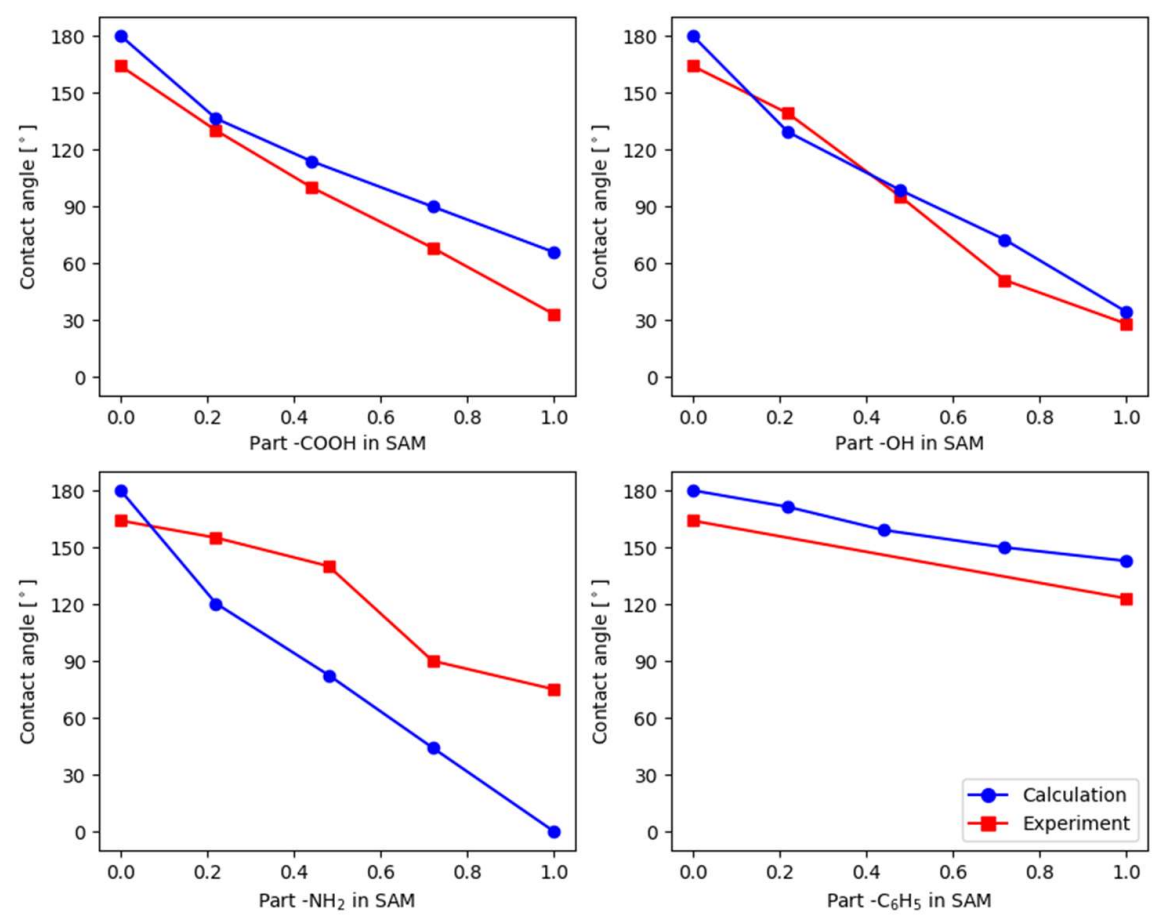

Figure 7. Comparison of experimental and predicted contact angles for a range of self assembled monolayers with mixed composition of $-\mathrm{CH}_{3}$ and the functional group indicated in each graph. 

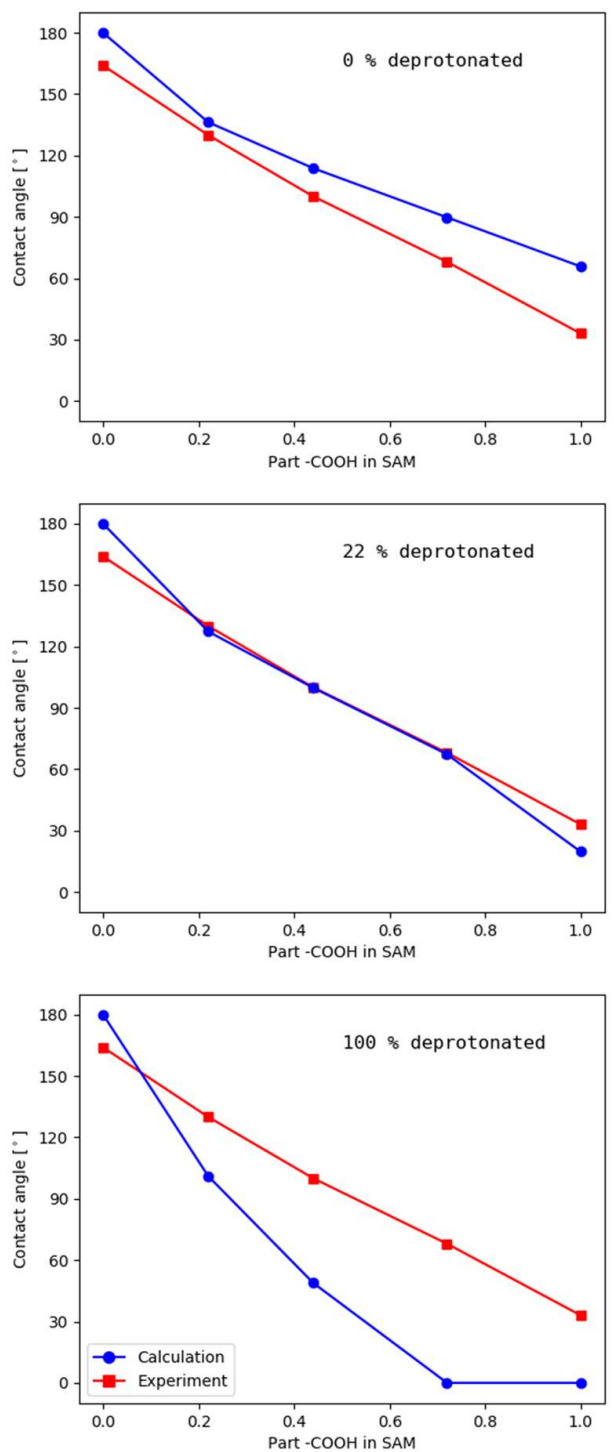

Figure 8. Comparison of experimental and predicted contact angle as a function of the degree of deprotonation in the -COOH SAM. $22 \%$ was the predicted degree of deprotonation from our mean field model at ionic strength of $0.1 \mathrm{M}$. 


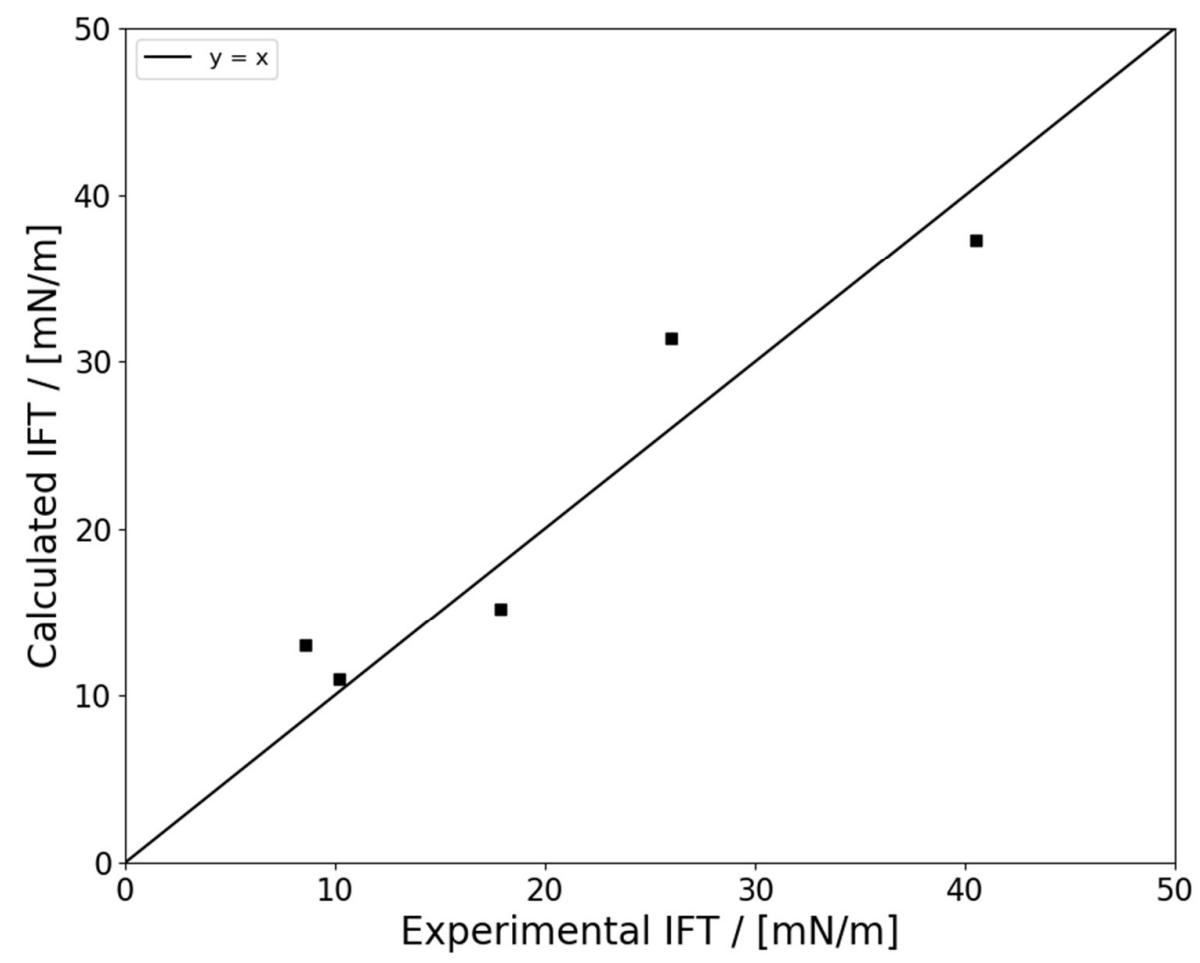

Figure 9. Comparison of the predicted and experimental solid-liquid interfacial free energies for PDMS and a range of liquids ${ }^{25}$.

\section{References}

1. (a) Tadros, M. E.; Hu, P.; Adamson, A. W., Adsorption and contact angle studies: I. Water on smooth carbon, linear polyethylene, and stearic acid-coated copper. J. Colloid Interface Sci. 1974, 49 (2), 184-195; (b) Hu, P.; Adamson, A. W., Adsorption and contact angle studies: II. Water and organic substances on polished polytetrafluoroethylene. J. Colloid Interface Sci. 1977, 59 (3), 605-614; (c) Xie, X.; Morrow, N. R., Contact angles on quartz induced by adsorption of heteropolar hydrocarbons. J. Adhes. Sci. Technol. 1999, 13 (10), 1119-1135; (d) Kwok, D. Y.; Neumann, A. W., Contact angle interpretation in terms of solid surface tension. Colloids Surf., A 2000, 161 (1), 31-48; (e) Ameri, A.; Kaveh, N. S.; Rudolph, E. S. J.; Wolf, K. H.; Farajzadeh, R.; Bruining, J., Investigation on Interfacial Interactions among Crude Oil-Brine-Sandstone Rock-CO2 by Contact Angle Measurements. Energy Fuels 2013, 27 (2), 1015-1025.

2. Giuffre, A. J.; Hamm, L. M.; Han, N.; De Yoreo, J. J.; Dove, P. M., Polysaccharide chemistry regulates kinetics of calcite nucleation through competition of interfacial energies. Proc. Natl. Acad. Sci. U. S. A. 2013, 110 (23), 9261-9266. 
3. Teng, H. H.; Dove, P. M.; Orme, C. A.; De Yoreo, J. J., Thermodynamics of calcite growth: Baseline for understanding biomineral formation. Science 1998, 282 (5389), 724-727.

4. Brown, K., Mineral Scaling in Geothermal Power Production. United Nations University Geothermal Training Programme: United Nations University Geothermal Training Programme, 2013; Vol. 39.

5. Haarberg, T.; Jakobsen, J. E.; Østvold, T., The Effect of Ferrous Iron on Mineral Scaling During Oil Recovery. Acta Chem. Scand. 1990, 44, 907-915.

6. Good, R. J.; Girifalco, L. A., A Theory for Estimation of Surface and Interfacial Energies. III. Estimation of Surface Energies of Solids From Contact Angle Data. The Journal of Physical Chemistry 1960, 64 (5), 561-565.

7. $\quad$ Freeman, C. L.; Hu, Q.; Nielsen, M. H.; Tao, J.; De Yoreo, J. J.; Harding, J. H., Surface Selectivity of Calcite on Self-Assembled Monolayers. The Journal of Physical Chemistry C 2013, 117 (10), 5154-5163.

8. Espinosa, J. R.; Vega, C.; Sanz, E., Ice-Water Interfacial Free Energy for the TIP4P, TIP4P/2005, TIP4P/Ice, and mW Models As Obtained from the Mold Integration Technique. The Journal of Physical Chemistry C 2016, 120 (15), 80688075.

9. Schimka, L.; Harl, J.; Stroppa, A.; Grüneis, A.; Marsman, M.; Mittendorfer, F.; Kresse, G., Accurate surface and adsorption energies from many-body perturbation theory. Nat. Mater. 2010, 9 (9), 741-744.

10. Andersson, M. P.; Bennetzen, M.; Klamt, A.; Stipp, S. L. S., First-principles Prediction of Liquid/Liquid Interfacial Tension. J. Chem. Theory Comput. 2014, 10 (8), 3401-3408.

11. Kuna, J. J.; Voitchovsky, K.; Singh, C.; Jiang, H.; Mwenifumbo, S.; Ghorai, P. K.; Stevens, M. M.; Glotzer, S. C.; Stellacci, F., The effect of nanometre-scale structure on interfacial energy. Nat. Mater. 2009, 8 (10), 837-842.

12. Ahlrichs, R.; Bar, M.; Haser, M.; Horn, H.; Kolmel, C., Electronic-structure calculations on workstation computers - the program system TURBOMOLE. Chem. Phys. Lett. 1989, 162 (3), 165-169.

13. Becke, A. D., Density-functional exchange-energy approximation with correct asymptotic behavior. Phys. Rev. A 1988, 38 (6), 3098-3100.

14. Perdew, J. P., Density-functional approximation for the correlation energy of the inhomogeneous electron gas. Phys. Rev. B: Condens. Matter 1986, 33 (12), 8822-8824.

15. Schafer, A.; Horn, H.; Ahlrichs, R., Fully optimized contracted gaussianbasis sets for atoms Li to Kr. J. Chem. Phys. 1992, 97 (4), 2571-2577.

16. Klamt, A.; Schüürmann, G., COSMO - A New Approach to Dielectric Screening in Solvents with Explicit Expressions for the Screening Energy and its Gradient. J. Chem. Soc., Perkin Trans. 2 1993, (5), 799-805.

17. Klamt, A.; Eckert, F.; Arlt, W., COSMO-RS: An Alternative to Simulation for Calculating Thermodynamic Properties of Liquid Mixtures. In Annual Review of Chemical and Biomolecular Engineering, Vol 1, 2010; Vol. 1, pp 101-122.

18. Eckert, F.; Klamt, A. COSMOtherm Version C3.0, Release 13.01, C3.0, Release 13.01; COSMOlogic GmbH \& Co. KG, Leverkusen, Germany: 2013.

19. (a) Lardge, J. S.; Duffy, D. M.; Gillan, M. J., Investigation of the Interaction of Water with the Calcite (10.4) Surface Using Ab Initio Simulation. J. Phys. Chem. $C$ 2009, 113 (17), 7207-7212; (b) Sakuma, H.; Andersson, M. P.; Bechgaard, K.; 
Stipp, S. L. S., Surface Tension Alteration on Calcite, Induced by Ion Substitution. J. Phys. Chem. C 2014, 118 (6), 3078-3087.

20. Hegner, M.; Wagner, P.; Semenza, G., Ultralarge atomically flat templatestripped Au surfaces for scanning probe microscopy. Surf. Sci. 1993, 291 (1), 3946.

21. Love, J. C.; Estroff, L. A.; Kriebel, J. K.; Nuzzo, R. G.; Whitesides, G. M., SelfAssembled Monolayers of Thiolates on Metals as a Form of Nanotechnology. Chem. Rev. (Washington, DC, U. S.) 2005, 105, 1103.

22. Brugnara, M. https://imagej.nih.gov/ij/plugins/contact-angle.html. 23. Andersson, M. P.; Olsson, M. H. M.; Stipp, S. L. S., Predicting pKa and stability of organic acids and bases at an oil-water interface Langmuir 2014, 30 (22), 6437-6445.

24. Baio, J. E.; Weidner, T.; Brison, J.; Graham, D. J.; Gamble, L. J.; Castner, D. G., Amine terminated SAMs: Investigating why oxygen is present in these films. J. Electron Spectrosc. Relat. Phenom. 2009, 172 (1-3), 2-8.

25. Mondal, S.; Phukan, M.; Ghatak, A., Estimation of solid-liquid interfacial tension using curved surface of a soft solid. Proceedings of the National Academy of Sciences 2015, 112 (41), 12563-12568.

26. Kosmulski, M., Surface Charging and Points of Zero Charge. CRC Press: 2009.

27. Goebel, A.; Lunkenheimer, K., Interfacial Tension of the Water/n-Alkane Interface. Langmuir 1997, 13 (2), 369-372. 